\title{
Mining social networks for local search and location-based recommender systems
}

\author{
Fabio Gasparetti ${ }^{1}$ - Damianos Gavalas ${ }^{2} \cdot$ Sergio Ilarri ${ }^{3} \cdot$ Francesco Ricci $^{4} \cdot{\text { Zhiwen } \mathrm{Yu}^{5}}^{5}$ \\ Published online: 6 June 2019 \\ (C) Springer-Verlag London Ltd., part of Springer Nature 2019
}

\section{Introduction and motivation}

Location-based services (LBSs) are software-level services that use location data in order to provide interesting and useful content to users or other services. The widespread usage of smartphones and wearable devices has made available large amounts of spatio-temporal data (e.g., geolocation, motion and environmental sensors). This new reality opens up compelling opportunities and raises challenges related to the automatic discovery and interpretation of data in pervasive environments. For instance, context-aware recommender systems (CARS) aggregate situational and environmental information about people, places and activities to satisfy immediate needs and offer enriched, situation-aware content, services and experiences. Popular applications are tourist tour planning and music recommender systems.

Fabio Gasparetti

gaspare@dia.uniroma3.it

Damianos Gavalas

dgavalas@ aegean.gr

Sergio Ilarri

silarri@unizar.es

Francesco Ricci

francesco.ricci@unibz.it

Zhiwen Yu

zhiwenyu@nwpu.edu.cn

1 Roma Tre University, Rome, Italy

2 University of the Aegean, Aegean, Greece

3 Aragon Institute of Engineering Research (I3A), University of Zaragoza, Zaragoza, Spain

4 Free University of Bozen-Bolzano, Bozen-Bolzano, Italy

5 Northwestern Polytechnical University, Xi'an, Shaanxi, China
While contextual factors quickly became the key of success of these pervasive applications, information related to user interests and preferences as well as social signals have not yet been adequately capitalized. The massive adoption of social applications, including social network services (e.g. Facebook and Twitter), collaborative tagging systems (e.g. Flickr and Delicious) and online communities (e.g. Foursquare and Yelp) gathers a wealth of social interactions between users, or between users and shared resources (e.g., points of interest, movies). Social local search and recommendation often refers to the search and recommendation paradigms affected by explicit or inferred social signals. The former are identified in the user's personal circle of friends, relatives or colleagues (egocentric network); the latter arise from groups of users that share common interests and behaviors (sociocentric network), even if no explicit ties bind them. Within this context, techniques employed for data and text mining, social network analysis and community detection, sentiment analysis and opinion mining have the chance to generate more accurate recommendations and personalized services. For instance, they can help us to understand more of the users' collective behavior by clustering similar users with respect to their interests, preferences and activities; or by recognizing knowledge experts, namely, users that are generally more capable than others in finding out relevant content and that therefore could play the role of trustworthy opinion sources.

The aim of this special issue is to explore recent advances in Local Search (LS) and Location-based Recommender Systems (LRS) focusing on the value, impact and implications of the analysis of social signals to alleviate information and interaction overload by filtering the most attractive and relevant content. This special issue has solicited original research contributions from academia and industry in the form of theoretical foundations, experimental and methodological developments, comparative analyses, descriptive surveys, experiments and case studies in the field. 


\section{Submissions, review process and summary of contributions}

The special issue of the Springer's Personal and Ubiquitous Computing on "Mining Social Networks for Local Search and Location-based Recommender Systems" has attracted 18 submissions from five continents (Africa, Asia, Central \& South America, Europe, and Oceania). The authors of the submitted papers are affiliated with institutions in Argentina, Australia, Austria, Brazil, China, India, Italy, Japan, Mexico, Spain, Switzerland, and Tunisia. Following a rigorous review process, five papers (acceptance rate 28\%) have been finally selected for inclusion in the special issue. Each paper received at least three reviews from independent experts. The accepted papers cover a wide spectrum of topics relevant to the special issue.

The first paper in the special issue, entitled "LOOKER: A Mobile, Personalized Recommender System in the Tourism Domain based on Social Media User-Generated Content" (contributed by Sondess Missaoui, Faten Kassem, Marco Viviani, Alessandra Agostini, Rim Faiz and Gabriella Pasi), introduces LOOKER, a mobile recommender system for tourism and travel-related services that considers the variability of interests and opinions of travelers. LOOKER implements a Content-Based Filtering strategy based on a multi-layer user profile, where the layers, representing distinct travel-related service categories, are modeled via language models defined on the basis of user-generated tourism-related content diffused in social media. The usefulness and usability of the LOOKER mobile application has been demonstrated through user studies.

The second manuscript, entitled "Point of Interest Recommendation Based on Social and Linked Open data" (authored by Giuseppe Sansonetti) proposes a personalized recommender system for Points of interest (POIs) located near the user's current position, which makes also use of Linked Open Data (LOD). In order to build and keep updated the profiles of the users' interests, the system monitors both their activity on social network services and their explicit feedback. LOD is exploited for enhancing the POI representation with additional information useful for the ranking. The performance of the POI recommender (with respect to its perceived accuracy) has been validated through an empirical evaluation.

In a similar scenario, the paper entitled "Exploiting Semantics for Context-Aware Itinerary Recommendation" (co-authored by Alessandro Fogli and Giuseppe Sansonetti) combines different techniques to perform context-aware personalized recommendation of itineraries with related multimedia content. Specifically, a graphbased structure represents the potential venues and their relevant attributes. By means of user-generated data, the mean residence time of each venue is also estimated. User profiles are built by a combination of explicit and implicit feedback techniques. LinkedGeoData is also considered for collecting additional data and providing personalized itinerary recommendations. The experimental evaluation has demonstrated the benefits of the proposed recommender in terms of accuracy and additional metrics (e.g., novelty and serendipity).

The fourth paper, entitled "Twitter-based Traffic Delay Detection based on Topic Propagation Analysis using Railway Network Topology" (by Yuanyuan Wang, Panote Siriaraya, Yukiko Kawai and Toyokazu Akiyama), proposes a Twitter-based railway delay detection method based on topic propagation analysis of geo-tagged tweets between railway stations. The key objective is to discover delay events and to predict train delays due to traffic accidents. The performance of the proposed method has been evaluated on datasets derived from Twitter with the actual delay information from stations and routes in the Tokyo area, Japan.

The last paper, titled "Recommender System for Learning Objects Based in the Fusion of Social Signals, Interests and Preferences of Learner Users in Ubiquitous e-Learning Systems" (co-authored by Alessandro da S. Dias and Leandro K. Wives), presents a recommendation approach for Learning Objects (LOs) in ubiquitous e-learning systems. The developed recommendation approach fuses data related to (a) user choices on social learning networks, and (b) social signals, interests and preferences of learners. Based on the fused data, it seeks to find the users that are more similar to the active user, in order to generate more accurate recommendations. The proposed approach has been experimentally evaluated, demonstrating significant higher prediction accuracy than baseline approaches.

Acknowledgments We would like to thank the authors of all the submitted papers for having considered this special issue and the Personal and Ubiquitous Computing journal as a potential publication venue for their research results. We would like to thank especially the authors of the accepted papers for their effort in revising and improving their work (occasionally, several times) in response to reviewers' comments. Many thanks also go to the PUC Editor-in-Chief Prof. Peter Thomas for his trust, guidance and support. The guest editors would also like to thank the support of some projects that fund their research activities in relation to the topics of the special issue, such as the project TIN2016-78011-C43-R (AEI/FEDER, UE) and the Government of Aragon (Group Reference T35 17D, COSMOS group) with the co-funding of Feder 2014-2020 "Construyendo Europa desde Aragon". Last but not least, we would like to thank the many anonymous reviewers who ensured the highest possible quality of the accepted papers and made this special issue possible.

Publisher's note Springer Nature remains neutral with regard to jurisdictional claims in published maps and institutional affiliations. 